\title{
Chronic photoperiodic
} manipulation induced chronodisruption upregulates HSP60 during early proatherogenic remodeling in thoracic aorta of C57BL/6J mice

\author{
Kavita Shirsath, Apeksha Joshi, Aliasgar Vohra and Ranjitsinh Devkar * (D)
}

\begin{abstract}
Background: Circadian disruption is often associated with aggravation of atherosclerosis; however, the pathophysiological mechanisms underlying atherogenic initiation in normolipidemic diet remains unclear. Most of the studies done for understanding circadian disruption induced atherosclerosis have been carried out in murine model of hyperlipidemia induced atherosclerosis. The present study investigates pro-atherogenic events in response to chronic photoperiodic manipulation induced chronodisruption (PMCD) in C57BL/6J mice fed with laboratory chow diet.

Results: The results were compared with atherogenic initiation induced by high fat high fructose (HFHF) diet. The combined effects of HFHF and PMCD on atherogenic initiation were also investigated for possible synergy of both variants. The HFHF and HFHF+PMCD groups recorded increments in body weight gains and serum lipid parameters (TC, TG, LDL-cholesterol, VLDL) and a decrement in HDL-cholesterol as compared to the control group. However, PMCD group recorded body weight gain similar to that of the control group, but the serum lipid parameters (TG and VLDL) were significantly elevated and the HDL levels were lowered. However, prominent hypertrophic remodeling, higher collagen deposition, and elastin derangement, along with endothelial dysfunction, its activation, and macrophage infiltration, were observed in thoracic aorta of all the three experimental groups. But the mRNA and immunoblots of heat shock protein 60 (HSP60) in thoracic aorta was found to be maximum in PMCD followed by HFHF and HFHF+PMCD groups.

Conclusion: Laboratory chow feeding coupled with photoperiodic manipulation mediated chronodisruption overexpress HSP60 that in turn plays a central role in PMCD mediated pro-atherogenic remodeling in thoracic aorta of C57BL/6J mice.
\end{abstract}

Keywords: Atherosclerosis, HSP60, Chronodisruption, Vascular remodeling, High fat high fructose diet

\footnotetext{
* Correspondence: rv.devkar-zoo@msubaroda.ac.in

Department of Zoology, Faculty of Science, The Maharaja Sayajirao University

of Baroda, Sayajigunj, Vadodara, Gujarat 390 002, India
}

\section{Springer Open}

(- The Author(s). 2021 Open Access This article is licensed under a Creative Commons Attribution 4.0 International License, which permits use, sharing, adaptation, distribution and reproduction in any medium or format, as long as you give appropriate credit to the original author(s) and the source, provide a link to the Creative Commons licence, and indicate if changes were made. The images or other third party material in this article are included in the article's Creative Commons licence, unless indicated otherwise in a credit line to the material. If material is not included in the article's Creative Commons licence and your intended use is not permitted by statutory regulation or exceeds the permitted use, you will need to obtain permission directly from the copyright holder. To view a copy of this licence, visit http://creativecommons.org/licenses/by/4.0/. 


\section{Background}

Intrinsic circadian clock is known to regulate synchrony of physiological processes in the body. Disturbance in biological rhythm due to the long working hours, shift jobs, and transcontinental travel is strongly associated with metabolic disorders. Epidemiological studies had demonstrated that shift workers comprise of a higher risk group for cardiovascular manifestations (Hermansson et al., 2019; Scheer, Hilton, Mantzoros, \& Shea, 2009). A cross-sectional study with night shift workers had suggested association of shift work with increased arterial stiffness that mark early atherogenic change (Jankowiak et al., 2016). Another observational study with factory workers had associated photoperiodic shifts with cardiovascular mortality (Knutsson, Hammar, \& Karlsson, 2004). Clinical studies had shown that acute cardiovascular events follow diurnal pattern indicating involvement of circadian clock components (Muller et al., 1985; Willich, 1990). In this regard, deletion or mutation of various circadian genes in murine models of atherosclerosis had been observed to promote plaque formation (Anea et al., 2009; Somanath et al., 2011). Western diet-induced lesion formation in aortic root was reported to be aggravated in ApoE*3-Leiden.CETP mice subjected to alternating lightdark conditions (Schilperoort et al., 2020). Thus, circadian disruption or photoperiodic alterations is an addition to the risk factors for atherosclerosis. Because of its multifactorial nature, the pathogenic mechanism underlying the atherogenic initiation need absolute clarity but till date, many facets remain unexplored.

Atherosclerosis is primarily associated with hyperlipidemia; however, majority of patients have been reported to have cholesterol levels within the normal range, suggesting a pathogenic mechanism independent of lipid levels (Fernandez-Friera et al., 2017; Ridker et al., 2008; Sniderman et al., 1980). Under such circumstances, the auto-antigenic role of heat shock protein 60 (HSP60) has emerged as a plausible explanation for the atherogenic initiation (Grundtman \& Wick, 2011). Serum levels of soluble HSP60 and autoreactive antibodies have been strongly correlated with atherosclerotic plaque severity (Xu et al., 1993; Xu et al., 1999; Xu et al., 2000). Also, ectopic expression of HSP60 in endothelial cells exposed to classical atherogenic risk factors has been observed (Amberger et al., 1997; Hochleitner et al., 2000; Jakic, Buszko, Cappellano, \& Wick, 2017; Kreutmayer et al., 2011). Simultaneous expression of adhesion molecules in stressed endothelium facilitates the sub-endothelial infiltration of leukocytes, thus promoting inflammation. In this context, accumulation of HSP60-autoreactive T cells has been reported to be the first event during atherogenic initiation in human aorta (Almanzar et al., 2012). Similarly, intravenous injection of bacterial lipopolysaccharides (LPS) in normocholesterolemic rabbits had upregulated intracellular adhesion molecule-1 (ICAM-1) and HSP60 expression in the arteries fulfilling the prerequisite for autoreactive $\mathrm{T}$ cell recruitment (Seitz, Kleindienst, $\mathrm{Xu}$, \& Wick, 1996). These changes are often accompanied by vascular dysfunction causing detrimental effects on the vascular tone leading to arterial stiffness. The infiltration of mononuclear cells in the vascular wall also causes inflammation contributing to the overall progression of the disease.

Atherogenic risk factors such as bacterial LPS, inflammatory cytokines, cigarette smoke extract, fluid shear stress, chlamydial infection, and hypertension have been associated with HSP60 induction in vascular endothelium (Amberger et al., 1997; Hochleitner et al., 2000; Jakic et al., 2017; Kreutmayer et al., 2011; Kreutmayer et al., 2013). Two cross-sectional studies with clinically healthy young males and females showed increased intima-media thickening that correlated with $\mathrm{T}$ cell reactivity against HSP60 wherein active and passive smoking were found to be major associated risk factors, respectively (Knoflach et al., 2003; Knoflach et al., 2009). High fat diet and atherogenic diet induced HSP60 expression have also been reported in atherosclerotic plaques of $\mathrm{ApoE}^{-1-}$ mice (Zhao et al., 2015). Thus, the upregulation of HSP60 in vascular wall has been acknowledged as a central mediator of atherogenic initiation, but the same has not been explored in the context of photoperiodic stress-induced atherosclerosis.

In light of these reports, the present study assesses atherogenic remodeling of thoracic aorta of C57BL/6J mice subjected to photoperiodic manipulation induced chronodisruption. High fat high fructose diet (HFHF) fed mice have been used as the disease positive control. A combination of HFHF+PMCD has also been put to scientific scrutiny to assess the possible synergistic effect on atherogenic transformation. Cause/s for pro-atherogenic changes in a normolipidemic condition is also investigated herein.

\section{Materials and methods}

\section{Animal experiments}

C57BL/6J male mice (20-22 g) of age 4-6 weeks were obtained from Advanced Center for Treatment, Research and Education in Cancer (ACTREC), Navi Mumbai, India. Mice were housed as 3-5 per cage and maintained as per the guidelines of the Committee for the Purpose of Control and Supervision of Experiments on Animals (CPCSEA) at the animal house of Department of Zoology, The Maharaja Sayajirao University of Baroda, Vadodara, Gujarat, India (827/GO/Re/S/04/CPCSEA). The animal experiment protocol (MSU-Z/IAEC/2/09-2017) was approved by the Institutional Animal Ethical Committee (IAEC), Department of Zoology, The Maharaja Sayajirao University of Baroda, and the experiments were conducted in accordance 
with the guidelines of CPCSEA. Mice were fed ad libitum with standard laboratory chow and water in rooms maintained in $12 \mathrm{~h}$ light $/ 12 \mathrm{~h}$ dark cycle at 23 to $25^{\circ} \mathrm{C}$ with humidity between $50-70 \%$. After 10 days of acclimatization, mice were randomly divided into four groups: control group $(n=6$, fed with standard chow and subjected to normal 12:12 light/dark cycle), high fat high fructose (HFHF) group $(n=10$, fed with high fat diet containing $35.3 \%$ fat content and $20 \%$ fructose in water and subjected to normal 12:12 light-dark cycle), the photoperiodic manipulation induced chronodisruption (PCMD) group ( $n=10$, fed with standard chow and subjected to photoperiodic manipulation induced chronodisruption), and the HFHF+PMCD group $(n=10$, fed with HFHF and subjected to PMCD). Mice undergoing photoperiodic manipulation induced chronodisruption were transferred from room 1 (7:00 to 19:00 h light/19:00 to 7:00 h dark period) to room 2 (11:00 to $23: 00 \mathrm{~h}$ dark/23: 00 to $11: 00 \mathrm{~h}$ light period) resulting in phase advance of 8 $\mathrm{h}$ (lights off at ZT4) and transferring back to room 1 resulting in a phase delay of $8 \mathrm{~h}$ (lights off at ZT20) (Fig. 1) on Mondays and Thursdays, respectively, at $10: 55 \mathrm{~h}$ (Kettner et al., 2015). Food intake and body weight were recorded every alternate day. At the end of 16 weeks, blood was collected from mice via retro-orbital sinus after overnight fasting and later were euthanized with mild isoflurane. The thoracic aortas were excised, fixed in $4 \%$ buffered paraformaldehyde (PFA), and embedded in paraffin using standard protocol for histochemical analysis. For gene expression studies, the tissue samples were snap frozen in liquid nitrogen and stored at $80^{\circ} \mathrm{C}$ until use. Serum was isolated from blood using standard protocol and was subjected to analysis of triglycerides (TG), total cholesterol (TC), very low density lipoprotein (VLDL), low density lipoprotein-cholesterol (LDL-Chol), and high density lipoprotein-cholesterol (HDL-Chol) using commercially available kits (Reckon Diagnostic kits, Vadodara, Gujarat, India).

\section{Histology and morphometric analysis}

PFA fixed thoracic aorta were dehydrated in a graded series of ethanol and embedded in paraffin blocks. Five to $6 \mu \mathrm{m}$ serial sections were cut using microtome and stained with hematoxylin-eosin (HXE; Sigma-Aldrich, USA). The sections were observed using Leica DMRB microscope (Leica Camera Inc., Germany) and images were captured. The intima-media thickness (IMT) and lumen area were measured using Image software $(\mathrm{NIH}$, Bethesda, USA) by an investigator blinded to experimental groups.

\section{Elastin auto-fluorescence}

Elastin auto-fluorescence was recorded from the HXE stained sections (Sawada \& Daugherty, 2018) using Floid imaging station (Life Technologies, USA). Elastin breaks, defined as discontinuity of an elastic fiber with boundaries at both sides clearly visible, were counted from the sections.

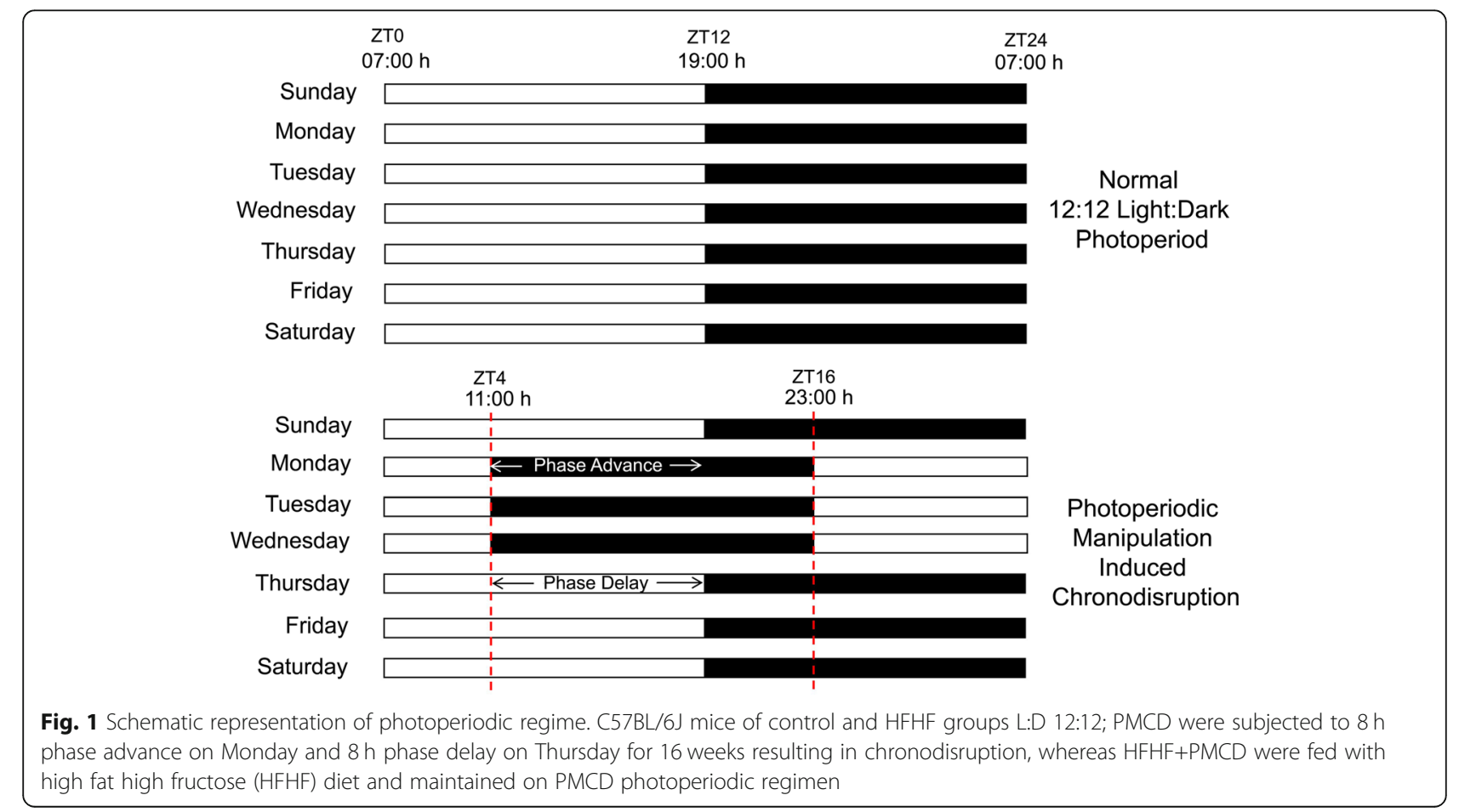




\section{Collagen staining}

Paraffin-embedded sections were de-paraffinized with xylene and rehydrated in a graded series of ethanol followed by staining with $0.1 \%$ direct red 80 (SigmaAldrich, USA) in saturated aqueous solution of picric acid for $1 \mathrm{~h}$ at room temperature (RT). Sections were observed under Leica DMRB microscope (Leica Camera Inc., Germany) and images were captured. Quantitative analysis of the images for collagen and elastin content was done using ImageJ software $(\mathrm{NIH}$, Bethesda, USA) (Borges, Taboga, \& Gutierrez, 2005; Vogel, Siebert, Hofmann, \& Frantz, 2015) and aortic stiffness was calculated as ratio of collagen to elastin.

\section{Immunohistochemical analysis}

Paraffin-embedded sections of thoracic aorta were subjected to immunohistochemical staining for detection of HSP60 or CD68. Briefly, 5-6 $\mu \mathrm{m}$ sections were cut and de-paraffinized in xylene followed by re-hydration using graded series of ethanol. Sections were subjected to antigen retrieval in sodium citrate buffer at $95^{\circ} \mathrm{C}$ for $20 \mathrm{~min}$ and endogenous peroxidases were masked with $3 \%$ hydrogen peroxide for $20 \mathrm{~min}$ in dark. Further, the sections were blocked with $1 \%$ fetal bovine serum for 30 min at RT followed by overnight incubation in primary antibody (HSP60 at 1:200; Cell Signaling Technology, USA, and CD68 at 1:100; Dako, Agilant, USA) at $4{ }^{\circ} \mathrm{C}$ in humidified chamber. After washing with PBS, sections were incubated with horseradish peroxidase (HRP)linked secondary antibody (Dako, Agilant, USA) for $1 \mathrm{~h}$ at RT. Thereafter, sections were washed thoroughly with PBS and DAB (3,3'-diaminobenzidine tetrahydrochloride) substrate (Dako, Agilant, USA) was added followed by counter-staining with hematoxylin. Sections were observed under Leica DMRB microscope (Leica Camera Inc., Germany) and images were captured. Quantification of positively stained regions was carried out using Fiji software (ImageJ, NIH, Bethesda, USA) (Schindelin et al., 2012).

\section{RNA isolation and quantitative RT-PCR}

Total RNA was isolated by homogenizing the tissue in Trizol reagent (Invitrogen, Thermo Fisher Scientific, USA) according to the manufacturer's protocol. One microgram of RNA was reverse transcribed to cDNA using iScript
cDNA synthesis kit (Bio-Rad Laboratories, USA). The cDNA was used for quantitative PCR analysis using Power Up SYBR Green Master Mix (Thermo Fisher Scientific, USA) in QuantStudio-3 Real-Time PCR System (Applied Biosystems, Thermo Fisher Scientific, USA). Expression levels were analyzed using $2^{-\Delta \Delta C T}$ method using GAPDH as endogenous control. Primer sequences specific for target mRNAs are listed in Table 1.

\section{Immunoblotting}

For extracting total protein lysate, tissue was homogenized in RIPA buffer (50 mM tris (pH 8.0), $150 \mathrm{mM}$ $\mathrm{NaCl}, 0.5 \%$ sodium deoxycholate, $0.1 \%$ sodium dodecyl sulfate, $1 \%$ triton-X-100) containing protease inhibitor cocktail (Sigma-Aldrich, USA) and $1 \mathrm{mM}$ PMSF, followed by incubation at $4{ }^{\circ} \mathrm{C}$ for $2 \mathrm{~h}$. The lysate was centrifuged at $10,000 \mathrm{rpm}$ at $4{ }^{\circ} \mathrm{C}$ for $20 \mathrm{~min}$ and resultant supernatant was subjected to protein estimation using Bio-Rad protein assay dye reagent (Bio-Rad Laboratories, USA). Twenty-five micrograms of protein was separated by SDS-PAGE and transferred onto PVDF membrane using Trans-Blot Turbo Transfer System (Bio-Rad Laboratories, USA). Membrane was blocked using 3\% bovine serum albumin in Tris-buffered saline (TBS) for $1 \mathrm{~h}$ at RT, followed by overnight incubation in anti-HSP60 (1:750; Cell Signaling Technology, USA) and anti- $\beta$-actin antibodies (1:1000; Invitrogen, Thermo Fisher Scientific, USA) at $4{ }^{\circ} \mathrm{C}$. Thereafter, the membrane was probed with HRP-labeled anti-rabbit secondary antibody (1:2000; Cell Signaling Technology, USA) for $1 \mathrm{~h}$ at RT. Expression of the immune-reactive proteins was detected using Clarity ${ }^{\mathrm{TM}}$ Western ECL Substrate (Bio-Rad Laboratories, USA) and chemiluminescence was recorded using iBright CL1000 Imaging Station (Invitrogen, Thermo Fisher Scientific, USA).

\section{Statistical analysis}

All the statistical analysis was carried out using GraphPad Prism 6.0. Differences between groups were compared by two-way ANOVA followed by Tukey's multiple comparison test. Results were expressed as mean \pm S.E.M. Differences were considered statistically significant at ${ }^{*} P \leq 0.05,{ }^{* *} P \leq 0.01$, and ${ }^{* * *} P \leq 0.001$.

Table 1 Primer sequences for quantitative PCR

\begin{tabular}{lll}
\hline Gene name & Forward primer $\left.\mathbf{( 5}^{\prime} \mathbf{\rightarrow} \mathbf{3}^{\prime}\right)$ & Reverse primer $\left(\mathbf{5}^{\prime} \boldsymbol{\rightarrow} \mathbf{3}^{\prime}\right)$ \\
\hline eNOS & TGGAAGGAAGTGCAGCAAA & GGCCAGTCTCAGAGCCATAC \\
$V C A M-1$ & CTGGGAAGCTGGAACGAAGT & GCCAAACACTTGACCGTGAC \\
$I C A M-1$ & GTGGTCGAAGGTGTTCTT & AAACAGGAACTTCCCGCCA \\
$M C P-1$ & TGACCCCAAGAAGGAATGGG & GACCTTAGGGCAGATGCAGTT \\
GAPDH & GTCGGTTGAACGGATTTGG & AGATGCCTGCTTCCCATTCT \\
\hline
\end{tabular}




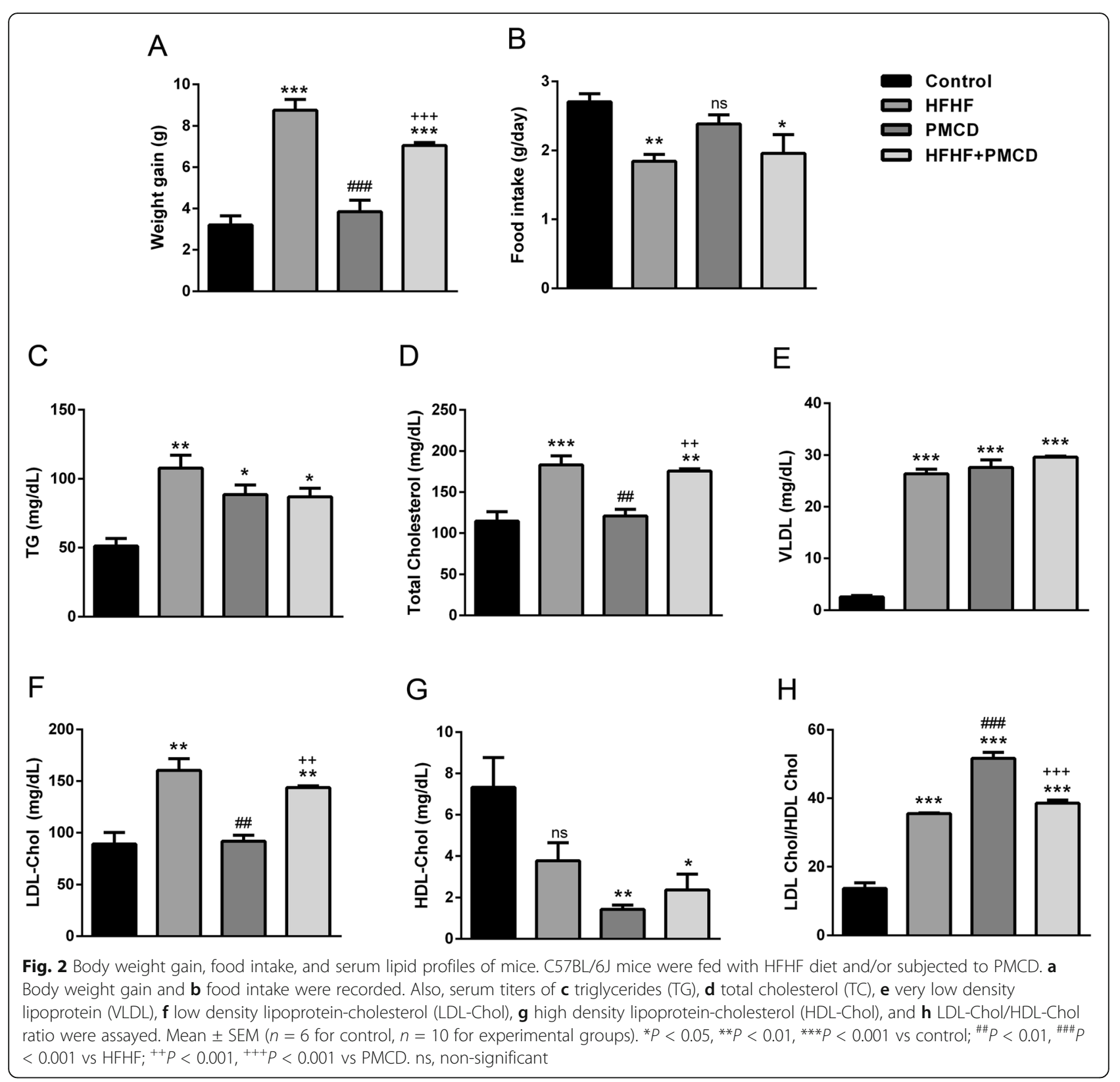

\section{Results}

HFHF and PMCD mediated alterations in body weight, food intake, and serum lipid profile

C57BL/6J mice were subjected to high fat high fructose (HFHF) diet, phase advance phase delay in photoperiod mediated chronodisruption (PMCD), and a combination of both variants (HFHF+PMCD). HFHF fed mice recorded significant increment in body weight and decrement in food intake as compared to control. PMCD group did not record any change in the said parameters and their values were comparable to that of control. HFHF+PMCD group recorded increment in body weight and decrement in food intake that was comparable to HFHF group (Fig. 2a, b). Parameters of the serum lipid profile viz. TG, TC, VLDL, LDL-Chol, and LDL-Chol-toHDL-Chol ratio were significantly elevated in HFHF and HFHF+PMCD groups. PMCD group recorded significant increment in TG, VLDL, and LDL-Chol-to-HDL-Chol ratio as compared to control, whereas HDL-Chol levels recorded a marked decrement in all the groups wherein the decrement was most significant in PMCD group (Fig. $2 \mathrm{c}-\mathrm{h})$.

HFHF and PMCD induces vascular remodeling in thoracic aorta

Microscopic evaluation of HXE stained sections of thoracic aorta revealed structural remodeling in intimal and medial regions in HFHF, PMCD, and HFHF+PMCD 


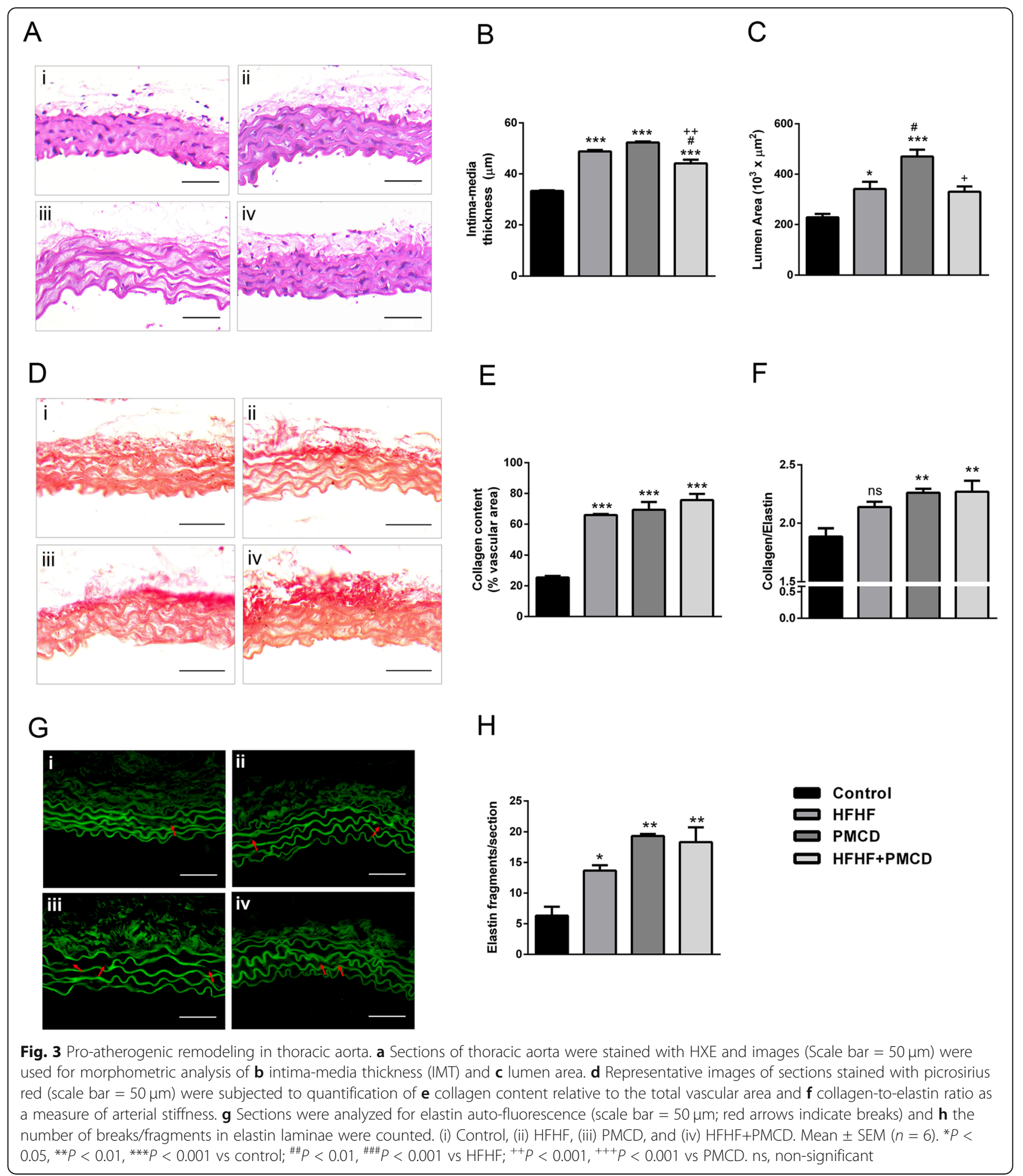

groups; the changes being most prominent in the PMCD group (Fig. 3a). The intima-media thickness (IMT) and luminal area of thoracic aorta showed increment in all the three groups with maximum significance recorded in the PMCD group (Fig. 3b, c). The collagen content recorded significant increments in all the three groups (Fig. 3e), whereas the collagen-to-elastin ratio recorded significant increments in the PMCD and HFHF+PMCD groups and an apparent, non-significant increase in the HFHF group (Fig. 3f). This parameter was assessed on the basis of thickening of the outer collagen observed in the picrosirius-stained sections (Fig. 3d). Auto-fluorescence of elastin in thoracic aorta 

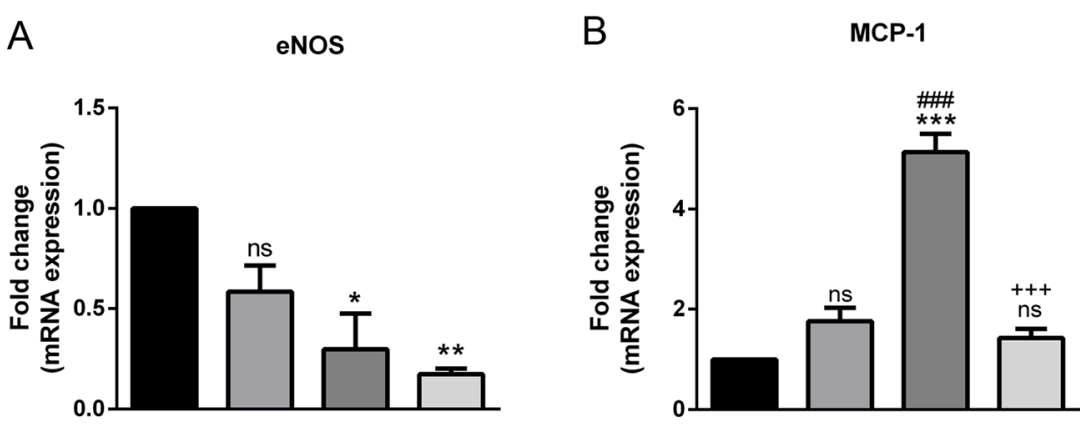

C

VCAM-1

D

ICAM-1
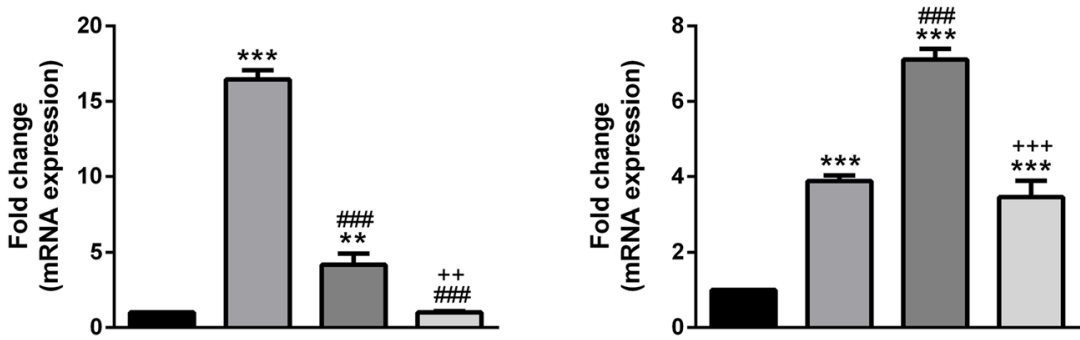

$\mathrm{E}$
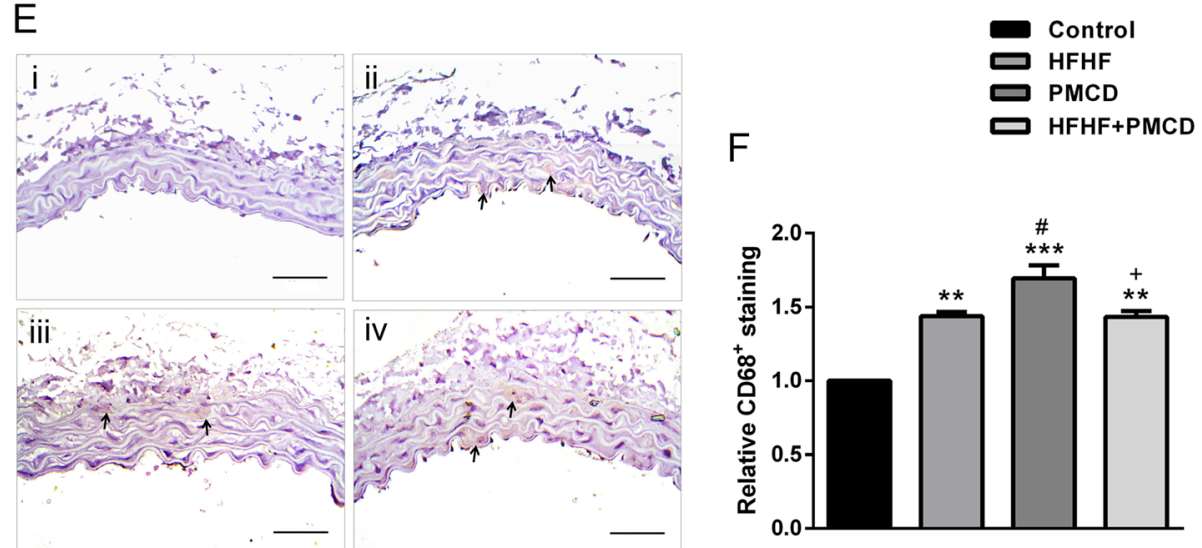

$\mathrm{F}$

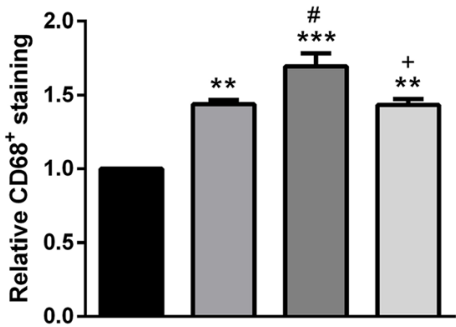

Fig. 4 Endothelial dysfunction and activation in thoracic aorta. Thoracic aorta were subjected to quantitative RT-PCR of a eNOS, b MCP-1, c VCAM-1, and d ICAM-1 mRNAs ( $n=3$ ). e Sections of thoracic aorta of (i) control, (ii) HFHF, (iii) PMCD, and (iv) HFHF+PMCD groups were immunostained for CD68 (macrophage marker) (scale bar $=50 \mu \mathrm{m}$; black arrows indicate CD68 ${ }^{+}$areas), and $\mathbf{d}$ quantification of the positive areas

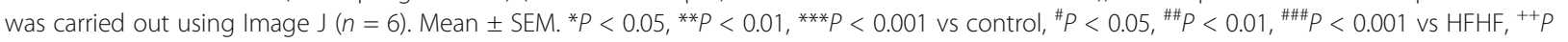
$<0.001,{ }^{+++} P<0.001$ vs PMCD. ns, non-significant

of experimental mice showed derangement in all the groups and with more prominence in the PMCD group (Fig. 3g). Increased number of elastin breaks were counted in all the treatment groups with highest value recorded in PMCD group (Fig. 3h).

\section{HFHF and PMCD induces endothelial dysfunction and atherogenic changes in thoracic aorta}

Endothelial nitric oxide synthase (eNOS) is a key marker for endothelial function and feeding with HFHF diet alone could induce only moderate (non-significant) decrement in eNOS mRNA. The decrement was significant in PMCD and HFHF+PMCD groups (Fig. 4a). Markers for endothelial activation and macrophage recruitment, viz. MCP-1, VCAM-1, and ICAM-1 were assessed in thoracic aorta. mRNA levels of the said genes were significantly higher in HFHF, PMCD, and HFHF+ PMCD groups (Fig. 4b). Immunolocalization of CD68 (macrophage marker) was significantly elevated in HFHF, PMCD, and HFHF+PMCD groups as evidenced by prominent positive staining of thoracic aorta (Fig. 4c, d). Overall, the said changes were more prominent in PMCD group. 

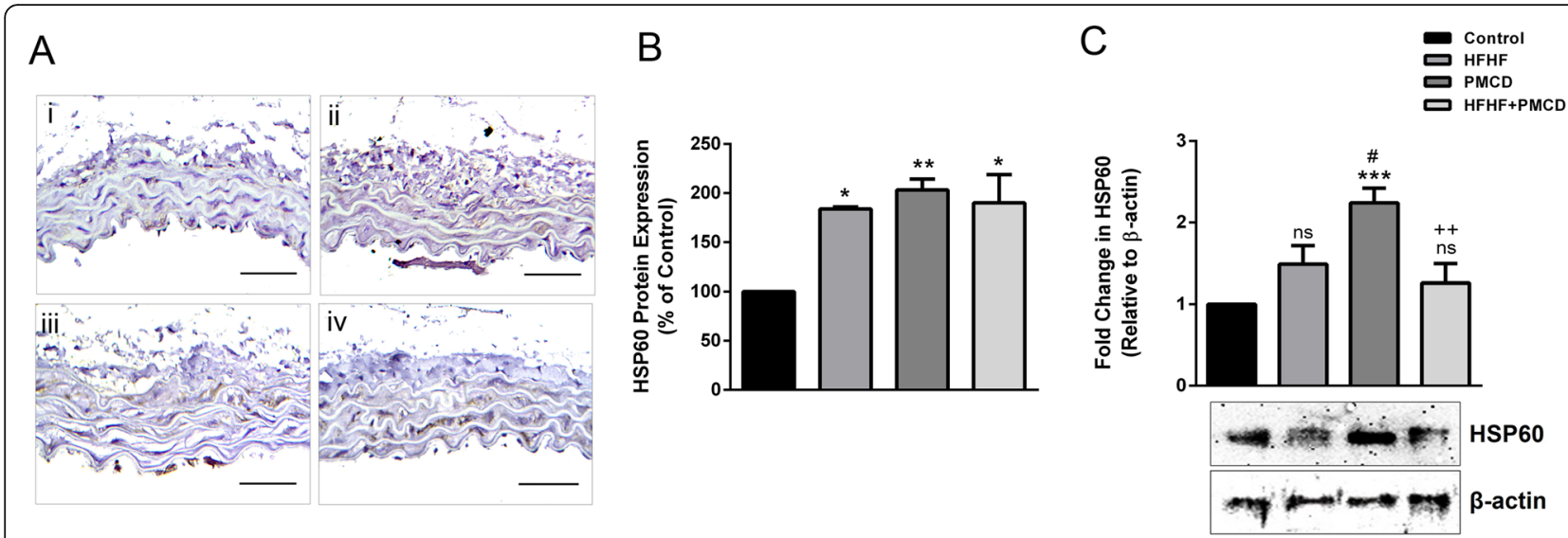

Fig. 5 HSP60 expression in thoracic aorta. a Sections of thoracic aorta of (i) control, (ii) HFHF, (iii) PMCD, and (iv) HFHF+PMCD groups were subjected to immunohistochemical staining of HSP60 (scale bar $=50 \mu \mathrm{m})$ and $\mathbf{b}$ positive stained regions were quantified by image J $(n=6)$. $\mathbf{c}$ HSP60 expression in thoracic aorta was also assessed by immunoblotting and the same was quantified by densitometry $(n=3)$. Mean \pm SEM. ${ }^{*} P$ $<0.05,{ }^{* *} P<0.01$ vs control, ${ }^{+} P<0.05$ vs PMCD. ns, non-significant

\section{HFHF and PMCD mediated upregulation of HSP60 in thoracic aorta}

Sections of thoracic aorta of HFHF, PMCD, and HFHF+ PMCD groups stained with anti-HSP60 antibody showed prominent positive immunostaining in all the three groups (Fig. 5a). Quantitative indices of the same were found to be significant (Fig. 5b). Further, immunoblots of HSP60 in thoracic aorta of the said groups showed similar trend of increment, but the values were nonsignificant in HFHF and PMCD+HFHF groups (Fig. 5c).

\section{Discussion}

The regulatory role of circadian clock system is well established in a number of physiological processes including pathophysiological changes in human diseases. Also, subtle variations in the compositions of a high fat diet have been implicated in atherosclerosis with varying degrees of pathophysiological manifestations (Getz \& Reardon, 2006; Lang, Hasselwander, Li, \& Xia, 2019; Ning et al., 2015; Santana et al., 2014; Schreyer, Wilson, \& LeBoeuf, 1998). However, development of cardiovascular ailments in normolipidemic condition has been implied to circadian dysregulationinduced physiological perturbations that lack clarity. In our study, the atherogenic changes resulting due to photoperiodic manipulations induced chronodisruption has been put to detailed investigation and the results have been compared with healthy (control) and HFHF diet fed C57BL/6J mice (HFHF). Also, HFHF diet fed mice subjected to circadian dysregulation (HFHF+PMCD) has been investigated for atherogenic pathophysiology.

Investigation of lipid profile of control and experimental groups revealed that the HFHF has a dominating presence in HFHF+PMCD group as the circulating titres of TG, TC, VLDL, and LDL-Chol were comparable in both the groups. However, the observed significant increment in
TG and VLDL, and significant decrement in HDL-Chol are the stand-out features of the photoperiodic manipulation induced chronodisruption. HDL-Chol has been extensively reported for its physiological relevance in lipid homeostasis via reverse cholesterol transport (Marques et al., 2018). In our study, though TC and LDL-Chol levels did not show significant increment in PMCD group, significant decrement in HDL-Chol forms the basis of proatherogenic manifestations observed herein. These observations are in agreement with a cross-sectional study conducted in Sweden that correlated shift work with increased triglycerides and decreased HDL-cholesterol levels (Karlsson, Knutsson, \& Lindahl, 2001).

Research reports on atherogenic diet fed mice/rats and high fat diet fed C57BL/6J mice have reported elevated lipid profiles and varying degrees of atherogenic changes in thoracic aorta (Cai, Miao, Xie, Lu, \& Su, 2005; Santana et al., 2014). Also, previous studies in our laboratory had shown atherogenic diet induced derangement in thoracic media and intima layers (Jadeja, Thounaojam, Jain, Devkar, \& Ramachandran, 2012; Patel, Desai, Gajaria, Devkar, \& Ramachandran, 2013; Thounaojam, Jadeja, Salunke, Devkar, \& Ramachandran, 2012). Similar changes were observed in the present study following feeding of HFHF diet wherein significant increment in IMT and lumen area corroborates our findings. Exposure of HFHF fed mice to photoperiodic manipulation induced chronodisruption also showed a similar remodeling of thoracic aorta. However, the said changes in thoracic aorta have not been reported in conditions of experimentally induced chronodisruption in mice fed with laboratory chow diet. Derangement of collagen and elastin fibers are the key events during atherogenic vascular remodeling and have been reported in our previous studies (Jadeja et al., 2012; Thounaojam et al., 
2012) and in thoracic aorta of HFHF diet fed mice in both HFHF and HFHF+PMCD groups. However, proatherogenic vascular remodeling observed in thoracic aorta of PMCD group is a significant finding because these mice were subjected to photoperiodic phase advance and phase delay along with laboratory chow diet. Photoperiodic regimes of Schilperoort et al. (2020) had resulted in aggravation of atherosclerosis in ApoE*3-Leiden.CETP mice, but the mice fed were maintained on a Westerntype diet. Another study by Anea et al. (2009) had reported pro-atherogenic vascular remodeling in Bmal1 knockout and Clock mutant mice maintained in constant darkness but these mice are naturally hyperlipidemic. In this regard, our study provides prima facie evidence on the role of photoperiodic manipulation induced chronodisruption as a causative agent for atherosclerosis without feeding atherogenic/high fat diet.

Pointing evidences on pro-atherogenic changes were obtained in the form of significant decrement in eNOS and significantly elevated atherogenic markers (MCP-1, VCAM-1, and ICAM-1) and macrophage marker (CD68) that confirmed endothelial dysfunction and subsequent atherogenic changes in all three experimental groups. These set of observations are in agreement with reports on high fat diet/atherogenic diet mediated induction of atherosclerosis (Jadeja et al., 2012; Lang et al., 2019; Thounaojam et al., 2012). Enhanced monocyte recruitment in lesions of ApoE*3-Lesion.CETP mice subjected to photoperiodic alterations has been reported, but diet induced hyperlipidemia was not ruled out as a contributing factor (Schilperoort et al., 2020). Herein, the aggravated response observed in PMCD group in terms of macrophage infiltration further supports the atherogenic progression in light of photoperiodic manipulation induced chronodisruption even in absence of dietary atherogenic stimuli.

Expression of cell adhesion molecules in proatherogenic vascular endothelium has been associated with HSP60 upregulation (Seitz et al., 1996) and our observations are in agreement with these reports. HSP60 upregulation suggests elevated levels of physiological stress in thoracic aorta (Hochleitner et al., 2000) that is comparable with smokers and hypertensive patients without conditions of hypercholesterolemia (Almanzar et al., 2012). Herein, we hypothesize that the pro-atherogenic changes observed in PMCD mice in spite of near normal levels of TC and LDL-Chol are attributable to photoperiod mediated stress and upregulation of HSP60 in thoracic aorta coupled with significantly low levels of HDL-Chol.

Taken together, our study highlights the role of HSP60 in inducing PMCD mediated pro-atherogenic changes, but a combination of HFHF and PMCD (HFHF+PMCD) does not result in synergistic symptoms. Overall, the findings hint at a possible underlying mechanism of atherosclerosis in normolipidemic patients.

\section{Abbreviations}

HSP60: Heat shock protein 60; HFHF: High fat high fructose;

PMCD: Photoperiodic manipulation induced chronodisruption

\section{Acknowledgements}

KS has acknowledged University Grant Commission (UGC), New Delhi, India, for providing fellowship (UGC-RGNF). Technical helps in various capacities rendered by Prof. Rajesh Singh (Biochemistry), Prof. Vihas Vasu (Zoology), and Dr. Kishore Rajput (Botany), MSU Baroda, are duly acknowledged.

\section{Authors' contributions}

K.S. and R.D. conceptualized the idea and designed the study. K.S., A.J., and A.V. performed in vivo experimentation. K.S. analyzed the data, whereas K.S. and R.D. interpreted the data and drafted the manuscript. All authors approve the final version of the manuscript.

\section{Funding}

The work was funded under Core Research Grant (SERB EMR/2015/002001) of Science and Engineering Research Board (SERB), New Delhi, India, to RD.

\section{Availability of data and materials}

All data generated or analyzed during this study are included in this published article.

\section{Ethics approval and consent to participate}

The animal experiment protocol (MSU-Z/IAEC/2/09-2017) was approved by the Institutional Animal Ethical Committee (IAEC), Department of Zoology, The Maharaja Sayajirao University of Baroda, and the experiments were conducted in accordance with the guidelines of the Committee for the Purpose of Control and Supervision of Experiments on Animals (CPCSEA).

\section{Consent for publication}

Not applicable

\section{Competing interests}

The authors declare that they have no competing interests.

Received: 29 May 2020 Accepted: 2 January 2021

Published online: 21 January 2021

\section{References}

Almanzar, G., Ollinger, R., Leuenberger, J., Onestingel, E., Rantner, B., Zehm, S., .. Wick, G. (2012). Autoreactive HSP60 epitope-specific T-cells in early human atherosclerotic lesions. Journal of Autoimmunity, 39, 441-450.

Amberger, A., Maczek, C., Jurgens, G., Michaelis, D., Schett, G., Trieb, K., ... Wick, G. (1997). Co-expression of ICAM-1, VCAM-1, ELAM-1 and Hsp60 in human arterial and venous endothelial cells in response to cytokines and oxidized low-density lipoproteins. Cell Stress \& Chaperones, 2, 94-103.

Anea, C. B., Zhang, M., Stepp, D. W., Simkins, G. B., Reed, G., Fulton, D. J., \& Rudic, R. D. (2009). Vascular disease in mice with a dysfunctional circadian clock. Circulation, 119, 1510-1517.

Borges, L. F., Taboga, S. R., \& Gutierrez, P. S. (2005). Simultaneous observation of collagen and elastin in normal and pathological tissues: Analysis of Sirius-redstained sections by fluorescence microscopy. Cell and Tissue Research, 320, 551-552.

Cai, G. J., Miao, C. Y., Xie, H. H., Lu, L. H., \& Su, D. F. (2005). Arterial baroreflex dysfunction promotes atherosclerosis in rats. Atherosclerosis, 183, 41-47.

Fernandez-Friera, L., Fuster, V., Lopez-Melgar, B., Oliva, B., Garcia-Ruiz, J. M., Mendiguren, J., ... Sanz, J. (2017). Normal LDL-cholesterol levels are associated with subclinical atherosclerosis in the absence of risk factors. Journal of the American College of Cardiology, 70, 2979-2991.

Getz, G. S., \& Reardon, C. A. (2006). Diet and murine atherosclerosis. Arteriosclerosis, Thrombosis, and Vascular Biology, 26, 242-249.

Grundtman, C., \& Wick, G. (2011). The autoimmune concept of atherosclerosis. Current Opinion in Lipidology, 22, 327-334.

Hermansson, J., Boggild, H., Hallqvist, J., Karlsson, B., Knutsson, A., Nilsson, T., .. Gillander Gadin, K. (2019). Interaction between shift work and established coronary risk factors. International Journal of Occupational and Environmental Medicine, 10, 57-65.

Hochleitner, B. W., Hochleitner, E. O., Obrist, P., Eberl, T., Amberger, A., Xu, Q., .. Wick, G. (2000). Fluid shear stress induces heat shock protein 60 expression 
in endothelial cells in vitro and in vivo. Arteriosclerosis, Thrombosis, and vascular Biology, 20, 617-623.

Jadeja, R. N., Thounaojam, M. C., Jain, M., Devkar, R. V., \& Ramachandran, A. V. (2012). Clerodendron glandulosum.Coleb leaf extract attenuates in vitro macrophage differentiation and expression of VCAM-1 and P-selectin in thoracic aorta of atherogenic diet fed rats. Immunopharmacology and Immunotoxicology, 34, 443-453.

Jakic, B., Buszko, M., Cappellano, G., \& Wick, G. (2017). Elevated sodium leads to the increased expression of HSP60 and induces apoptosis in HUVECS. PLOS One, 12, e0179383.

Jankowiak, S., Backe, E., Liebers, F., Schulz, A., Hegewald, J., Garthus-Niegel, S., ... Latza, U. (2016). Current and cumulative night shift work and subclinical atherosclerosis: Results of the Gutenberg health study. International Archives of Occupational and Environmental Health, 89, 1169-1182.

Karlsson, B., Knutsson, A., \& Lindahl, B. (2001). Is there an association between shift work and having a metabolic syndrome? Results from a population based study of 27,485 people. Occupational and Environmental Medicine, 58, 747-752.

Kettner, N. M., Mayo, S. A., Hua, J., Lee, C., Moore, D. D., \& Fu, L. (2015). Circadian dysfunction induces leptin resistance in mice. Cell Metabolism, 22, 448-459.

Knoflach, M., Kiechl, S., Kind, M., Said, M., Sief, R., Gisinger, M., .. Wick, G. (2003). Cardiovascular risk factors and atherosclerosis in young males: ARMY study (atherosclerosis risk-factors in male youngsters). Circulation, 108, 1064-1069.

Knoflach, M., Kiechl, S., Penz, D., Zangerle, A., Schmidauer, C., Rossmann, A., ... Wick, G. (2009). Cardiovascular risk factors and atherosclerosis in young women: Atherosclerosis risk factors in female youngsters (ARFY study). Stroke, 40, 1063-1069.

Knutsson, A., Hammar, N., \& Karlsson, B. (2004). Shift workers' mortality scrutinized. Chronobiology International, 21, 1049-1053.

Kreutmayer, S., Csordas, A., Kern, J., Maass, V., Almanzar, G., Offterdinger, M., . Wick, G. (2013). Chlamydia pneumoniae infection acts as an endothelial stressor with the potential to initiate the earliest heat shock protein 60 dependent inflammatory stage of atherosclerosis. Cell Stress \& Chaperones, 18 , 259-268.

Kreutmayer, S. B., Messner, B., Knoflach, M., Henderson, B., Niederegger, H., Bock, G., ... Bernhard, D. (2011). Dynamics of heat shock protein 60 in endothelia cells exposed to cigarette smoke extract. Journal of Molecular and Cellular Cardiology, 51, 777-780.

Lang, P., Hasselwander, S., Li, H., \& Xia, N. (2019). Effects of different diets used in diet-induced obesity models on insulin resistance and vascular dysfunction in C57BL/6 mice. Scientific Reports, 9, 19556.

Marques, L. R., Diniz, T. A., Antunes, B. M., Rossi, F. E., Caperuto, E. C., Lira, F. S., \& Goncalves, D. C. (2018). Reverse cholesterol transport: Molecular mechanisms and the non-medical approach to enhance HDL cholesterol. Frontiers in Physiology, 9, 526

Muller, J. E., Stone, P. H., Turi, Z. G., Rutherford, J. D., Czeisler, C. A., Parker, C., et al. (1985). Circadian variation in the frequency of onset of acute myocardial infarction. The New England Journal of Medicine, 313, 1315-1322.

Ning, B., Wang, X., Yu, Y., Waqar, A. B., Yu, Q., Koike, T., ... Fan, J. (2015). Highfructose and high-fat diet-induced insulin resistance enhances atherosclerosis in Watanabe heritable hyperlipidemic rabbits. Nutrition \& Metabolism (London), 12, 30.

Patel, D., Desai, S., Gajaria, T., Devkar, R., \& Ramachandran, A. V. (2013). Coriandrum sativum L. seed extract mitigates lipotoxicity in RAW 264.7 cells and prevents atherogenic changes in rats. EXCLI Journal, 12, 313-334.

Ridker, P. M., Danielson, E., Fonseca, F. A., Genest, J., Gotto Jr., A. M., Kastelein, J. J., ... Group, J.S (2008). Rosuvastatin to prevent vascular events in men and women with elevated C-reactive protein. The New England Journal of Medicine, 359, 2195-2207.

Santana, A. B., de Souza Oliveira, T. C., Bianconi, B. L., Barauna, V. G., Santos, E. W., Alves, T. P., ... Lacchini, S. (2014). Effect of high-fat diet upon inflammatory markers and aortic stiffening in mice. BioMed Research International, 2014, 914102.

Sawada, H., \& Daugherty, A. (2018). Quantification of elastin fragmentation in the ascending aorta.

Scheer, F. A., Hilton, M. F. Mantzoros, C. S., \& Shea, S. A. (2009). Adverse metabolic and cardiovascular consequences of circadian misalignment. Proceedings of the National Academy of Sciences of the United States of America, 106, 4453-4458.

Schilperoort, M, van den Berg, R, Bosmans, L. A, van Os, B. W. Dolle, M. E. T, Smits, N. A. M., ... Kooijman, S. (2020). Disruption of circadian rhythm by alternating light-dark cycles aggravates atherosclerosis development in APOE*3-Leiden.CETP mice. Journal of Pineal Research, 68, e12614.

Schindelin, J., Arganda-Carreras, I., Frise, E., Kaynig, V., Longair, M., Pietzsch, T., ... Cardona, A. (2012). Fiji: An open-source platform for biological-image analysis. Nature Methods, 9, 676-682.

Schreyer, S. A., Wilson, D. L., \& LeBoeuf, R. C. (1998). C57BL/6 mice fed high fat diets as models for diabetes-accelerated atherosclerosis. Atherosclerosis, 136, 17-24.

Seitz, C. S., Kleindienst, R., Xu, Q., \& Wick, G. (1996). Coexpression of heat-shock protein 60 and intercellular-adhesion molecule- 1 is related to increased adhesion of monocytes and T cells to aortic endothelium of rats in response to endotoxin. Laboratory Investigation, 74, 241-252.

Sniderman, A., Shapiro, S., Marpole, D., Skinner, B., Teng, B., \& Kwiterovich Jr., P. O. (1980). Association of coronary atherosclerosis with hyperapobetalipoproteinemia [increased protein but normal cholesterol levels in human plasma low density (beta) lipoproteins]. Proceedings of the National Academy of Sciences of the United States of America, 77, 604-608.

Somanath, P. R., Podrez, E. A., Chen, J., Ma, Y., Marchant, K., Antoch, M., \& Byzova, T. V. (2011). Deficiency in core circadian protein Bmal1 is associated with a prothrombotic and vascular phenotype. Journal of Cellular Physiology, 226, 132-140.

Thounaojam, M. C., Jadeja, R. N., Salunke, S. P., Devkar, R. V., \& Ramachandran, A $V$. (2012). Sida rhomboidea.Roxb aqueous extract down-regulates in vivo expression of vascular cell adhesion molecules in atherogenic rats and inhibits in vitro macrophage differentiation and foam cell formation. Immunopharmacology and Immunotoxicology, 34, 832-843.

Vogel, B., Siebert, H., Hofmann, U., \& Frantz, S. (2015). Determination of collagen content within picrosirius red stained paraffin-embedded tissue sections using fluorescence microscopy. MethodsX, 2, 124-134.

Willich, S. N. (1990). Epidemiologic studies demonstrating increased morning incidence of sudden cardiac death. The American Journal of Cardiology, 66, 15G-17G.

Xu, Q., Kiechl, S., Mayr, M., Metzler, B., Egger, G., Oberhollenzer, F., ... Wick, G. (1999). Association of serum antibodies to heat-shock protein 65 with carotid atherosclerosis : Clinical significance determined in a follow-up study. Circulation, 100, 1169-1174.

Xu, Q., Schett, G., Perschinka, H., Mayr, M., Egger, G., Oberhollenzer, F., ... Wick, G. (2000). Serum soluble heat shock protein 60 is elevated in subjects with atherosclerosis in a general population. Circulation, 102, 14-20.

Xu, Q., Willeit, J., Marosi, M., Kleindienst, R., Oberhollenzer, F., Kiechl, S., ... Wick, G. (1993). Association of serum antibodies to heat-shock protein 65 with carotid atherosclerosis. Lancet, 341, 255-259.

Zhao, Y., Zhang, C., Wei, X., Li, P., Cui, Y., Qin, Y., ... Gao, Y. (2015). Heat shock protein 60 stimulates the migration of vascular smooth muscle cells via tolllike receptor 4 and ERK MAPK activation. Scientific Reports, 5, 15352.

\section{Publisher's Note}

Springer Nature remains neutral with regard to jurisdictional claims in published maps and institutional affiliations.

\section{Submit your manuscript to a SpringerOpen ${ }^{\circ}$ journal and benefit from:}

- Convenient online submission

- Rigorous peer review

- Open access: articles freely available online

- High visibility within the field

- Retaining the copyright to your article

Submit your next manuscript at $>$ springeropen.com 